Documentos PRESEEA de investigación

Guía PRESEEA

de estudio

del presente narrativo

Silvana Guerrero González

y Silvana Arriagada Anabalón
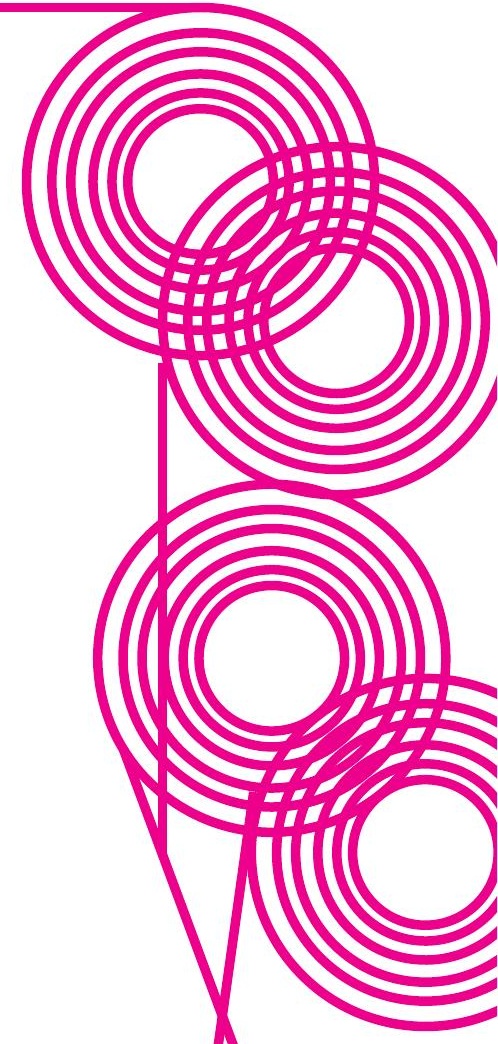
Reservados todos los derechos. Queda prohibida, salvo excepción prevista en la ley, cualquier forma de reproducción, distribución, comunicación pública y transformación de esta obra sin contar con la autorización de los titulares de propiedad intelectual. La infracción de los derechos mencionados puede ser constitutiva de delito contra la propiedad intelectual (arts. 270 y sigs., Código Penal). El Centro Español de Derechos Reprográficos (www.cedro.org) vela por el respeto de los citados derechos.

Esta publicación ha sido financiada por el Grupo de investigación Lingüística de la variación espacial y social (LIVARES) y por el Proyecto para el estudio sociolingüístico del español de España y Amémica (PRESEEA).

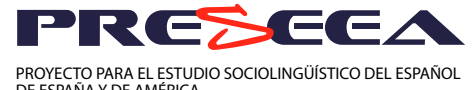
PROYECTO PARA EL ESTUDIO SOCIOLINGÜISTICO DEL ESPAÑOL
DEESPANNAY DE AMERICA
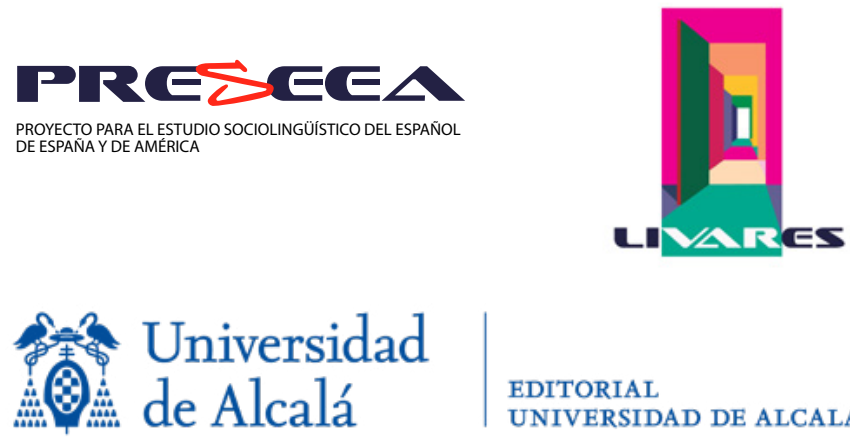

EDITORIAL

UNIVERSIDAD DE ALCALÁ

C Silvana Guerrero González y Silvana Arriagada Anabalón

Editan: Proyecto para el estudio sociolingüístico del español de España y

América (PRESEEA)

Colegio San José de Caracciolos

C/Trinidad, $5 \cdot 28801$ Alcalá de Henares (Madrid, España)

Web: https://preseea.linguas.net/

Editorial Universidad de Alcalá, 2021

Plaza de San Diego, s/n.ํ • 28801, Alcalá de Henares (España)

Web: uah.es 


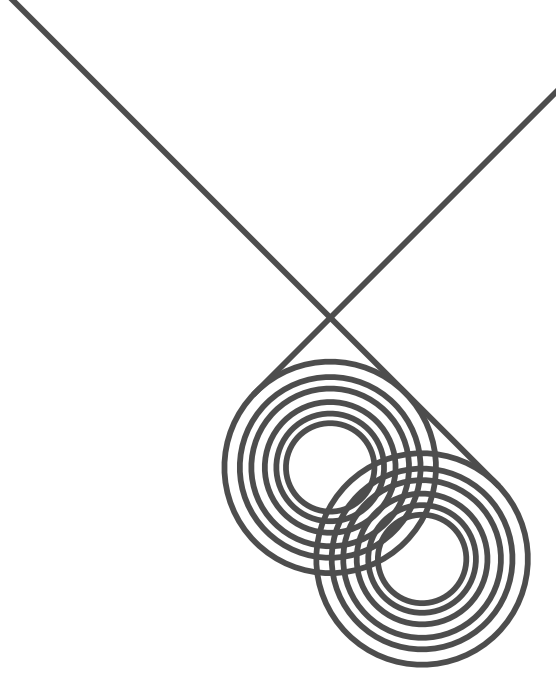

\section{Guía PRESEEA de estudio del presente narrativo}

Silvana Guerrero González (Universidad de Chile - Chile) siguerrero@u.uchile.cl

Silvana Arriagada Anabalón (Pontificia Universidad Católica de Chile - Chile)

sbarriagada@uc.cl 


\section{Índice}

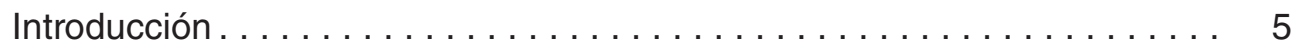

Bases metodológicas para el estudio coordinado del presente narrativo. .......................... 7

PRESEEA_PRESENTE NARRATIVO. Ficha para codificación

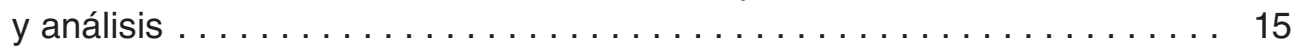

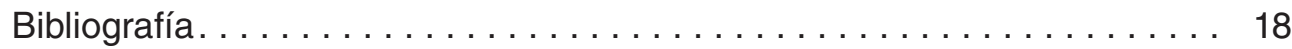




\title{
Introducción
}

\begin{abstract}
E
objetivo general del estudio del 'presente narrativo' con los materiales del PRESEEA es indagar en su variación geolingüística y sociolingüística en el ámbito hispanohablante. Con este propósito se intenta desarrollar una línea de trabajo basada en un fenómeno lingüístico propio de las narraciones y compatible con los eventos referidos en pasado. Se trata del uso del presente morfológico para referir al pasado, cuyo empleo está dado por el propósito comunicativo y por el contexto de aparición. Es el presente narrativo el mecanismo que nos interesa particularmente en tanto fenómeno variable presente en la secuencia narrativa, pero de manera específica, en narraciones de experiencia personal provenientes de las entrevistas sociolingüísticas.
\end{abstract}

La pretensión inicial de esta Guía es intentar dar respuesta a dos preguntas generales, mediante su aplicación sistemática: a) por qué hay individuos que emplean más los presentes narrativos que otros y b) qué funciones cumple el presente narrativo dentro de las narraciones de experiencia personal en las entrevistas sociolingüísticas. Se seguirán las pautas metodológicas para los grupos PRESEEA, de las que adelantamos algunas aquí:

- Todos los investigadores que formen parte del grupo deberán utilizar los materiales del corpus PRESEEA como fuente primaria de datos para sus análisis y realizar sobre ellos distintos tipos de estudios, tanto cualitativos como cuantitativos, que proporcionen información sobre la frecuencia de aparición del fenómeno variable en estudio, así como las relaciones existentes entre este y la incidencia de factores sociales -edad, sexo y nivel de instrucción-, geográficos y lingüísticos que podrían favorecer o no su aparición.

- Para abordar el presente narrativo, se han propuesto dos modalidades:

a) Tratamiento de las formas verbales para aludir al pasado como una variable en el sentido clásico

b) Tratamiento del presente narrativo como una variable en el sentido no clásico 
- Para el análisis y la codificación, se ha establecido una ficha general básica. En ella se han listado variables lingüísticas y no lingüísticas de estudio:

1. Individuo

2. Verbos

3. Persona verbal

4. Presencia o no de introductores sintáctico-discursivos ante los verbos

5. Sección de la narración donde aparece el presente narrativo

6. Posible efecto mecánico de aparecer o no en una secuencia de presentes narrativos

7. Factores sociales

8. Factores geográficos

- Se podrán realizar análisis generales, considerando las modalidades de trabajo $A$ o $B$, o estudios en profundidad, seleccionando alguno de los aspectos propuestos para determinar la variación del presente narrativo.

Los estudios que se emprendan dentro de PRESEEA_PRESENTE NARRATIVO partirán de la ficha común de codificación y de los criterios básicos de análisis, que podrán ser ampliados, matizados, etc. por los investigadores del grupo, a partir de los hallazgos que se vayan obteniendo. Con esto, las autoras de esta propuesta estamos convocando a otros investigadores a realizar sugerencias y observaciones que permitan avanzar hacia el estudio coordinado del presente narrativo.

Todos los documentos necesarios para trabajar sobre el presente narrativo en PRESEEA_PRESENTE NARRATIVO están disponibles en la página electrónica del proyecto (preseea.linguas.net). Las coordinadoras del grupo de investigación quedamos a disposición para aclarar cualquier duda y compartir información al respecto. 


\section{Bases metodológicas para el estudio coordinado del presente narrativo}

\section{¿Qué entendemos por presente narrativo?}

EI

estudio de las narraciones tiene una larga tradición en sociolingüística, desde Labov y Waletzky (1967) a Labov (2013), por mencionar dos trabajos especialmente relevantes. El recuento de hechos personales proporciona un contexto de gran interés para estudiar ciertos fenómenos que se ubican entre la morfosintaxis y el discurso. Como precisan Lastra y Martín Butragueño (2017), uno de estos fenómenos es precisamente el presente narrativo, ya materia de los trabajos de Wolfson $(1978,1979)$, pero sólidamente estudiado en español, sea en trabajos clásicos como los de Silva-Corvalán (1983, 2001), sea en análisis más recientes, como el de Guerrero González (2014), el de Lastra y Martín Butragueño (2017) o el de Guerrero et al. (2020).

El presente histórico corresponde a un uso particular de las formas de presente para hacer referencia a situaciones pasadas (miro por miré). Alarcos Llorach (1994: 156) señala que "se llama presente histórico al empleo, tanto en la narración escrita como en el vivo relato coloquial, de las formas de presente para aludir a hechos cronológicamente ocurridos en el pasado". Asimismo, los estudios sobre presente histórico concuerdan en que este uso particular del presente indicativo tiene efectos estilísticos en el discurso, dotándolo de mayor fuerza, viveza y dramatismo, y denominándolo, en consecuencia, "presente teatralizado" (cf. Bello 1841, Gili Gaya 1961, Chafe 1982, Calsamiglia y Tusón 1999, Guerrero González 2014, entre otros).

El presente histórico, al alternar con formas verbales en pretérito, conlleva mayores efectos de viveza y dramatismo, cumpliendo una función evaluativa dentro del relato (Silva-Corvalán 1983, 2001). A aquellos recursos evaluativos que cumplen la función de suspender la acción, Labov (1972) los denomina dispositivos de "evaluación externa" y los recursos que permiten preservar la continuidad dramática reciben el nombre de mecanismos de "evaluación interna." Al respecto, 
Silva-Corvalán (1983) plantea que el presente histórico corresponde a un mecanismo de evaluación interna, ya que actúa dentro de la cadena narrativa y se vincula con eventos que transforman la narración en reportable, por lo tanto, responde a una función evaluativa. Esta autora comprueba empíricamente que el empleo del presente histórico es más frecuente en la sección de complicación de la narración. Chafe (1982) señala que el presente histórico es un rasgo propio del involucramiento y asigna importantes marcas de vivacidad, y Calsamiglia y Tusón (1999) también lo ponen de relieve cuando destacan sus efectos de viveza dentro de la narración.

La Nueva Gramática de la Lengua Española (2009) incorpora la diferencia postulada entre los usos del presente histórico en discursos escritos y discursos orales, distinguiendo entre el "presente histórico", característico de las biografías y de las descripciones históricas, cuyo rasgo principal es la posibilidad de trasladar el punto del habla a un momento del pasado, y el "presente narrativo", propio de las narraciones y compatible con los eventos referidos en pasado. En suma, ambos casos consisten en valores trasladados del verbo - uso del presente morfológico para referir al pasado- cuya diferencia está dada por el propósito comunicativo y por el contexto de aparición. Es el presente narrativo el que nos interesa particularmente en tanto fenómeno variable en la secuencia narrativa.

Así descrito, el presente narrativo queda bien ejemplificado en (1), (2) y (3), tomados de relatos orales de entrevistas sociolingüísticas realizadas en el marco del PRESEEA de las variedades del español de Santiago de Chile, Caracas y Montevideo.

(1) [...] y loco / yo de repente veo que eso[s] se van con los dos tipos después se <sic/> / corren / pum / en la dirección que íbamos nosotros [...] (SCHI_M13_079).

(2) [...] le dice a mi hermano que va pasando <cita> mira / este / aguántame esto aquí / mientras yo voy / que mi mamá me está llamando </cita $>$ jay! ipara suerte de mi hermano aparece un policía secreto! / ¡mi hermano de catorce años! y le dice <cita> baja el papagayo </cita> por supuesto que yo me imagino que mi hermano identificó / si yo sabía que existía policía secreto / mi papá / mi hermano rápido supo que era policía secreto / entonces bajó el papagayo / el papagayo de [nombre propio] tenía una cruceta / lo que / yo no sé si tú sabes / una cruceta de / de / que eso se hace con gillette en la punta / bueno por esa razón se llevaron a mi hermano para la casilla de arriba / más arriba del molinito éste que me encantaba a mí / que había un caminito del molino se cogía una ca <palabra_cortada/> y se iba a la casilla (CARA_M33_103).

(3) sí mirá / hasta hace / hasta hace bastante un hasta hace un par de años por ejemplo eran hermosas porque de chicos nos juntábamos en la casa de mi tío / que vive ahí a dos cuadras de mi casa / que tiene una casa grandísima y $</$ sic $>$ 
invitaba a toda la familia y amigos y entonces eran como cuarenta personas y me dicen / y me encantaba que hubiera mucha gente/ se ponía música/ se bailaba / se comía de todo / uno / un menú muy variado / estaba precioso ¿viste? // ahora que está un poco enfermo (MONV_H12_006).

Como se observa en los ejemplos citados, una característica del presente narrativo es su potencial conmutación por la misma forma verbal en pretérito. Con esta base, podemos decir que en (1) se puede reemplazar veo por vi, se van por se fueron y corren por corrieron; en (2) dice por dijo y va pasando por iba pasando; y en (3) dicen por dijeron. Esta característica se debe a que, aunque el suceso reportado se posicione cronológicamente en el pasado, igualmente hace avanzar la acción. Esta observación es importante en la aplicación de esta Guía toda vez que en los relatos los narradores incorporan comentarios con formas verbales en presente que no necesariamente continúan con la descripción de la sucesión de hechos reportados, como sucede en (2) cuando el hablante señala por supuesto que yo me imagino.

\section{Preguntas de investigación}

Con la Guía de codificación del presente narrativo se intenta dar respuesta a dos preguntas generales. La primera implica cuestiones de orden cuantitativo, mientras que la segunda debe tener necesariamente un enfoque cualitativo: a) por qué hay individuos que emplean más los presentes narrativos que otros y b) qué funciones cumple dentro de las narraciones de experiencia personal en las entrevistas sociolingüísticas. Ambas preguntas están relacionadas, pues se vinculan con la eficiencia narrativa de las personas, sus propósitos comunicativos y con los recursos lingüísticos y discursivos que se eligen para ser narrativamente eficientes (Lastra y Martín Butragueño 2017).

Respecto de (a) y sobre la existencia de diferencias entre los individuos, caben al menos dos posibilidades: una, que las diferencias se deban a alguna característica social transversal. A ese propósito, esta Guía considera tres características básicas: nivel de estudios, edad y sexo de los individuos, con el propósito de explorar el efecto o la incidencia de los factores sociales - de manera independiente o en interacción- en el empleo del presente narrativo. Los hallazgos sobre este aspecto podrían contribuir a determinar cómo continuar la investigación de los agrupamientos sociales relacionados con el uso de este fenómeno. Otra posibilidad es que las diferencias se deban al desarrollo de habilidades personales o a historias de vida específicas que no necesariamente constituyen un reflejo de las características sociodemográficas del grupo al que se los adscribe. En este caso, se trata de variables externas, en la mayoría de las ocasiones, no controladas en la entrevista sociolingüística, por lo tanto, se sugiere verificar la centralidad de los individuos en una red social, por ejemplo. De esta manera, 
queda incorporada la posibilidad de indagar sobre si el peso de los individuos particulares es mayor al de las categorías sociales en las que se inscriben (Guerrero González 2014; Lastra y Martín Butragueño 2017).

En relación con (b) la bibliografía sugiere que los presentes narrativos en las narraciones orales de experiencia personal se emplean para contribuir a la reportabilidad de una narración. Este recurso evaluativo es uno de los tantos que permite que una historia se considere como "digna de contarse" (Labov 2013, Silva-Corvalán 2001, Guerrero González 2014).

\section{Propuesta metodológica}

La propuesta metodológica del presente narrativo en el corpus PRESEEA incluye dos modalidades básicas:

\section{A. Tratamiento de las formas verbales para aludir al pasado como una variable en el sentido clásico}

Esta alternativa de análisis comprende el fichaje tanto de los pretéritos como de los presentes narrativos, en tanto formas de aludir a hechos ocurridos en el pasado, es decir, tanto el pretérito como el presente narrativo se consideran variantes de una variable. En este sentido, ambas formas verbales tendrían 'igualdad funcional' en la medida en que permiten hacer referencia a un hecho pasado dentro una narración. La variabilidad consistiría en la posibilidad que tienen los hablantes de "elegir" uno de dichos valores o variantes como medio de justificación del carácter reportable del relato de experiencia personal. Al emplear el presente narrativo los sujetos le otorgan un valor especial a la narración, esto es, justificarla y dotarla de mayor dinamismo (Guerrero González 2014, Lastra y Martín Butragueño 2017).

\section{B) Tratamiento del presente narrativo como una variable en el sentido no clásico}

En este caso, solo se fichan los presentes narrativos, pero no los pretéritos que pudieran haberse intercambiado por presentes, dado su elevado número. Esto hace que el tratamiento de las variables no sea el clásico, pues no hay una variante de "aplicación" versus una de "no aplicación" de la variable dependiente.

Una vez escogida la modalidad de análisis ( $A \circ B$ ), se sugiere trabajar con una sola narración por entrevista, la más completa ${ }^{1}$, como sugiere Guerrero

\footnotetext{
${ }^{1}$ La narración más completa corresponde a la mejor desarrollada por cada informante, que es, básicamente, aquella que contiene la mayor cantidad de cláusulas narrativas en términos labovianos. De esta forma, se puede medir el mejor desempeño de cada uno de hablantes que conforman la muestra de estudio y, posteriormente, comparar sus desempeños narra-
} 
González (2014) o dejando de lado los inicios de las entrevistas —donde muchas veces la interacción es más esquemática-y las partes finales - fichando todos los casos de presente narrativo que aparecieran entre las páginas 6 y 25 de las transcripciones, como proponen Lastra y Martín Butragueño (2017).

Los aspectos que se sugiere considerar son los siguientes:

a) Individuos: sobre este ítem se sugiere evaluar si el empleo del presente narrativo responde a los hábitos particulares e individuales de cada hablante o al desarrollo de una entrevista.

b) Verbos: sobre este ítem se propone estudiar cuáles son las piezas verbales involucradas en el empleo del presente narrativo, incluyendo datos de frecuencias.

c) Persona verbal: en este caso, se debe considerar el análisis de los rasgos morfológicos asociados a la persona, con el propósito de determinar en cuáles de ellas se distribuyen las diferentes ocurrencias.

d) Presencia o no de introductores sintáctico-discursivos ante los verbos: sobre este ítem se propone indagar en la existencia de ciertos recursos que introducen los presentes narrativos, como conjunciones, adverbios, deícticos, entre otros. Un caso de común interés es el estudio de de repente + presente narrativo (ejemplo 1).

e) Sección de la narración donde aparece el presente narrativo: sobre este ítem se sugiere determinar la parte estructural de la narración -resumen, orientación, complicación, evaluación, resolución- en la que se emplean el presente narrativo.

f) El posible efecto mecánico de aparecer o no en una secuencia de presentes narrativos: sobre este ítem se propone indagar sobre la posición de aparición del presente narrativo, es decir, considerar si se trata de la primera aparición en un tramo de cláusulas narrativas o si existe un antecedente inmediato dentro del mismo tramo de cláusulas. Esto permite determinar si se trata de usos aislados o de organizaciones secuenciales.

g) Los factores sociales: sobre este ítem se sugiere estudiar la incidencia de los factores sexo, edad y nivel de estudios de los hablantes, por separado y en interacción.

tivos. Este criterio de selección permite controlar algunas variables intervinientes, como el cansancio y el desinterés de los sujetos, considerando la duración de la entrevista. 
h) Los factores geográficos: sobre este ítem, se sugiere estudiar el empleo del presente narrativo de manera contrastiva entre las diferentes variedades del español, intentando responder a las preguntas básicas propuestas en esta Guía.

Con el propósito de responder las preguntas básicas propuestas en esta Guía metodológica, el fenómeno debe abordarse tanto cuantitativa como cualitativamente.

A partir del ejemplo (4) se presentan apreciaciones acerca de cómo podrían considerarse algunos de los aspectos mencionados:

(4) cuando me dieron el balazo en el noventa // matan a una persona / entonces yo / cuando volteo así / y veo que me están apuntando ahí yo me tiro al suelo // me disparan la bala entra por la parte de acá atrás // un recorrido así / <ininteligible/> y baja la bala / quedó pegada al corazón y ahí está // me llevan al hospital // me meten al / al <siglas = [tac] $>$ TAC $</$ siglas $>$ / a la máquina esa grande / me dice el doctor <cita> va a estar tres semanas aquí mira // e / se rompió tanto su / se rompió tanto el pulmón / cuando entró la bala se rompió el pulmón cinco pulgadas / se quebró dos costillas / bajó la bala/ está pegada al corazón / se va mover la bala / tengo que esperar/ si no en seis semanas tengo que operarte $</$ cita $>$ / ¿qué crees que le dije yo al doctor? (MONR_H22_052).

En (4) el hablante incluye en presente narrativo, previo a la citas del doctor, los verbos voltear, ver, apuntar, tirarse, disparar, entrar, bajar, llevar, meter y decir. Como precisáramos antes, en este caso el uso de matan, en la primera línea, no hace avanzar el relato, por lo tanto, no debe considerarse. A partir de este ejemplo, se puede investigar con los presupuestos teórico-metodológicos de la modalidad A, según la cual se debe fichar los casos de presente narrativo y los de pretérito, para comparar sus distintos propósitos comunicativos y contextos de aparición. Si se prefiere realizar un estudio no clásico de la variable, se puede trabajar con la modalidad B.

También es clave que en (4) el presente narrativo aparece asociado a la voz del narrador, mientras que los casos de pretérito, como rompió y entró quedan vinculados a la reproducción de discurso ajeno. En otro orden, en los casos de presente narrativo, el informante incluye la primera y la tercera persona verbal. Asimismo, se trata de un fenómeno que aparece en la complicación del relato y, por último, en la medida de que se estudie una muestra más amplia, se puede verificar si la ocurrencia del presente narrativo es más propia de los hombres o de las mujeres de algún nivel educacional específico. 


\section{Plan de trabajo}

\section{Presentación PRESEEA_PRESENTE NARRATIVO}

La convocatoria de invitación para realizar estudios sobre el presente narrativo con los materiales del PRESEEA se encuentra disponible en la página electrónica del PRESEEA desde 2017.

Esta Guía surge siguiendo los acuerdos establecidos en la reunión PRESEEA realizada en el Congreso de Asociación de Lingüística y Filología de América Latina, ALFAL, celebrado en Bogotá en julio de 2017. Para su elaboración se tuvo en cuenta la Guía PRESEEA para la investigación lingüística.

\section{Estudios PRESEEA_PRESENTE NARRATIVO}

Los trabajos que han abordado el presente narrativo en el marco del PRESEEA son todavía escasos. Esto se debe a que la Guía metodológica es más bien reciente y solo en los últimos años se ha empezado a poner el foco en la ejecución coordinada de trabajos que aborden la caracterización de este fenómeno, cuyo empleo se ubica entre la morfosintaxis y el discurso, y que incluye, además, un fuerte carácter pragmático.

Sirvan de muestra de estudio el trabajo publicado por Guerrero González (2014), el expuesto por Lastra y Martin Butragueño (2017) y la publicación de Guerrero et al. (2020). Las tres investigaciones utilizan la modalidad de análisis B de esta Guía.

La Guía está sujeta a modificaciones y ajustes derivados de la experiencia de los investigadores.

3. Protocolo de coordinación

Se consideran tres aspectos fundamentales: 
- Convocatoria: están convocados todos los equipos PRESEEA interesados en el estudio del presente narrativo, para formar parte de este grupo de investigación.

- Coordinación de equipos de trabajo: entre los interesados se distribuirá la ficha de codificación, los criterios básicos de análisis y la bibliografía pertinente.

- Difusión de resultados: una vez que se avance en las investigaciones, se organizarán sesiones en coloquios y publicaciones monográficas que den cuenta de los resultados sobre la variación geolingüística y sociolingüística del presente narrativo en el ámbito hispanohablante. 


\section{PRESEEA_PRESENTE NARRATIVO Ficha para codificación y análisis}

PASO 1: elegir entre las modalidades $A$ y $B$

a) Tratamiento de las formas verbales para aludir al pasado como una variable en el sentido clásico

b) Tratamiento del presente narrativo como una variable en el sentido no clásico

PASO 2: análisis

Elegir una narración por entrevista o trabajar con la transcripción que va entre los minutos 6 y 25 de cada entrevista.

Unidad de análisis

Cláusula narrativa

Variable dependiente

Presente narrativo

Variables independientes (factores lingüísticos y no lingüísticos)

Análisis cuantitativo y cualitativo de los casos de empleo del presente narrativo.

Se sugiere realizar la cuantificación por individuo, con el propósito de disponer de una planilla de datos específicos que luego permitan realizar agrupaciones (por sexo, edad, nivel de instrucción, etc.) y, finalmente, llevar a cabo las pruebas estadísticas correspondientes. 


\section{Individuo}

a. Cuantificación de los presentes narrativos empleados por individuo

\section{Verbos}

a. Catálogo de verbos en presente narrativo

b. Cuantificación de verbos en presente narrativo por individuo

\section{Persona verbal}

a. Clasificación de empleo del presente narrativo por persona verbal

b. Cuantificación del presente narrativo por persona verbal por individuo

4. Presencia o no de introductores sintáctico-discursivos ante los verbos

a. Catálogo de introductores sintáctico-discursivos para el presente narrativo (entonces, de repente, $y$, etc.)

b. Cuantificación de introductores sintáctico-discursivos por individuo

5. Sección de la narración donde aparece el presente narrativo

a. Clasificación de los casos de presente narrativo por sección narrativa:

1 Resumen

2 Orientación

3 Complicación

4 Evaluación

5 Resolución

b. Cuantificación de los casos de presente narrativo por sección narrativa por individuo

6. Posible efecto mecánico de aparecer o no en una secuencia de presentes narrativos

a. Clasificación de la ubicación del presente narrativo por cláusula narrativa por individuo

\section{Factores sociales}

a. Cuantificación por sexo por individuo

1 Hombre

2 Mujer

b. Cuantificación por edad por individuo

120 a 34 años

235 a 54 años

355 años y más 
c. Cuantificación por nivel de instrucción por individuo

1 Estudios primarios

2 Estudios secundarios

3 Estudios superiores

\section{Factores geográficos}

a. Cuantificación que incluya de 1 a 7, o eligiendo entre 1 y 7 por variedades del español en los corpus PRESEEA por individuo

1 Valencia

2 Alcalá

3 Las Palmas

4 Granada

5 Málaga

6 Madrid (Distrito de Salamanca)

7 Madrid (Vallecas)

8 Monterrey

9 Santiago de Chile

10 San Juan de Puerto Rico

11 etc. O según las variedades elegidas para el estudio 


\section{Bibliografía}

Alarcos Llorach, Emilio. 1994. Gramática de la lengua española, Madrid, Espasa-Calpe.

Bello, Andrés. 1841. Análisis ideológica de los tiempos de la conjugación castellana: reproducción facsimilar de la edición Príncipe, Valparaíso, 1841, Caracas, Venezuela, Plan Cultural Caracas.

Calsamiglia, Helena y Amparo Tusón. 1999. Las cosas del decir. Manual de análisis del discurso, Barcelona, Ariel.

Chafe, Wallace. 1982. Integration and involvement in speaking, writing, and oral literature, en D. Tannen (ed.), Spoken and written language. Exploring orality and literacy, Norwood, NJ, Estados Unidos, Ablex: 35-53.

Guerrero González, Silvana. 2014. Una aproximación sociolingüística al empleo del presente histórico en narraciones de experiencia personal en hablantes de Santiago de Chile, RLA, Revista de Lingüística Teórica y Aplicada, 52, 2: 89-112.

Guerrero, Silvana, Javier González y Silvana Arriagada. 2020. Narrative present in the Spanish of Santiago, Chile Convergence and divergence with the Spanish variety from Mexico City, Spanish in Context, 17, 2: 341-361.

Labov, William. 2013. The Language of Life and Death. The Transformation of Experience in Oral Narrative, Cambridge, Cambridge University Press.

Labov, William y Joshua Waletzky. 1967. Narrative analysis: oral versions of personal narrative, en J. Helm (ed), Proceedings of the 1966 Annual Spring Meeting of the American Ethnological Sociey. Seattle - London, American Ethnological Society, University of Washington Press: $12-44$.

Lastra, Yolanda y Pedro Martín Butragueño. 2017. El presente narrativo en historias de experiencia personal en el Corpus sociolingüístico de la Ciudad de México, Ponencia XVIII Congreso Internacional de ALFAL, 24-28 julio de 2017, Bogotá, Colombia.

Moreno Fernández, Francisco. 1997. Metodología del «Proyecto para el Estudio Sociolingüístico del Español de España y de América», Trabajos de sociolingüística hispánica, Alcalá de Henares, Universidad de Alcalá: 137-166.

Real Academia Española. 2009. Nueva Gramática De La Lengua Española (NGLE), Madrid/ España, Espasa Calpe.

Silva-Corvalán, Carmen. 1983. Tense and aspect in oral Spanish narrative: context and meaning, Language, 59, 4: 760-780. 
Silva-Corvalán, Carmen. 2001. Análisis del discurso, en Sociolingüística y pragmática del español, Washington, Georgetown University Press: 192-237.

Wolfson, Nessa. 1978. A feature of performed narrative: the conversational historical present, Language in Society, 7, 2: 215-237.

Wolfson, Nessa. 1979. The conversational historical present alternation, Language, 55, 1 : 168-182. 